\title{
Reciprocity formulae for general Dedekind-Rademacher sums
}

\author{
by
}

R. R. Hall (York), J. C. Wilson (York) and D. Zagier (Bonn)

1. Introduction. Let

$$
\bar{B}_{1}(x)= \begin{cases}x-[x]-1 / 2 & (x \in \mathbb{R} \backslash \mathbb{Z}), \\ 0 & (x \in \mathbb{Z}) .\end{cases}
$$

If $b$ and $c$ are coprime integers the classical Dedekind sum $s(b, c)$ is defined by

$$
s(b, c)=\sum_{h(\bmod c)} \bar{B}_{1}\left(\frac{h}{c}\right) \bar{B}_{1}\left(\frac{b h}{c}\right) .
$$

Dedekind [5] introduced this sum in connection with the transformation formula for the Dedekind $\eta$-function and deduced from this his reciprocity formula

$$
s(b, c)+s(c, b)=-\frac{1}{4}+\frac{1}{12}\left(\frac{b}{c}+\frac{1}{b c}+\frac{c}{b}\right) .
$$

The Dedekind sum has been generalized, notably by Rademacher [9], who introduced the homogeneous sum $s(a, b ; c)$ in which the fraction $h / c$ in the left-hand Bernoulli function above becomes $a h / c$. Rademacher's reciprocity formula, which is not implied by Dedekind's, is (for $a, b, c$ pairwise coprime)

$$
s(a, b ; c)+s(b, c ; a)+s(c, a ; b)=-\frac{1}{4}+\frac{1}{12}\left(\frac{a}{b c}+\frac{b}{c a}+\frac{c}{a b}\right) .
$$

The standard reference for ordinary Dedekind sums is Rademacher and Grosswald [10]. Apostol [1], Carlitz [3] and Mikolás [7] defined sums involving higher order Bernoulli functions. The inhomogeneous and homogeneous sum become, respectively,

$$
S_{m, n}(b, c)=\sum_{h(\bmod c)} \bar{B}_{m}\left(\frac{h}{c}\right) \bar{B}_{n}\left(\frac{b h}{c}\right)
$$


and

$$
S_{m, n}(a, b ; c)=\sum_{h(\bmod c)} \bar{B}_{m}\left(\frac{a h}{c}\right) \bar{B}_{n}\left(\frac{b h}{c}\right),
$$

where $\bar{B}_{m}(x)$ denotes the periodic extension onto $\mathbb{R}$ of the $m$ th Bernoulli polynomial restricted to $[0,1)$ (cf. $\S 2$ ). The authors cited gave reciprocity formulae for their sums, and all such linear relations with polynomial coefficients were classified by Hall and Wilson [6]. It emerges that Mikolás's relations for homogeneous sums form a complete set. In this paper we show that Mikolás's relations extend to the generalized Dedekind-Rademacher sum given by (5) below, in which the variables are affected by certain linear shifts. In the case $m=n=1$, Berndt [2] obtained this relation, and there is a similar result with a slightly different formulation in Carlitz [4]. The theorem presented in this paper contains all the previous results in the literature about shifted sums. The original, rather more complicated proof was given by the first two authors. It is based on general identities involving Bernoulli polynomials which are apparently new, and is contained in Wilson [12]. The present formulation and proof are due to the third author.

2. Review of Bernoulli polynomials. The $m$ th Bernoulli polynomial is defined by

$$
B_{m}(u)=\sum_{n=0}^{m}\left(\begin{array}{c}
m \\
n
\end{array}\right) B_{n} u^{m-n} \quad\left(B_{n}=n \text {th Bernoulli number }\right),
$$

or equivalently by the generating function

$$
\sum_{m=0}^{\infty} B_{m}(u) \frac{X^{m-1}}{m !}=\frac{e^{u X}}{e^{X}-1} .
$$

Its most important property is the Fourier expansion formula

$$
\sum_{k \in \mathbb{Z}}^{\prime} \frac{\mathbf{e}(k u)}{k^{m}}=-\frac{(2 \pi i)^{m}}{m !} \bar{B}_{m}(u) \quad(u \in \mathbb{R}, m \geq 1) .
$$

Here $\sum^{\prime}$ means that $k=0$ is to be omitted (and, in the non-absolutely convergent case $m=1$, the sum to be interpreted as a Cauchy principal value), $\mathbf{e}(u)$ denotes $\exp (2 \pi i u)$ as usual, and $\bar{B}_{m}(u)$ is defined as the unique function which is periodic of period 1 and coincides with $B_{m}(u)$ on $[0,1)$ (except that we set $\bar{B}_{1}(u)=0$ for $\left.u \in \mathbb{Z}\right)$. To prove (2), one can simply check the case $m=1$ directly (the left-hand side is essentially the Taylor expansion of the logarithm function) and observe that the $B_{m}(u)$ are uniquely characterized by the properties

$$
B_{0}(u)=1, \quad B_{m}^{\prime}(u)=m B_{m-1}(u), \quad B_{m}(0)=B_{m}(1) \quad \text { for } m>1 .
$$


We will also need the so-called "distribution property" (Raabe's formula [8])

$$
\sum_{f(\bmod a)} \bar{B}_{m}\left(x+\frac{f}{a}\right)=a^{1-m} \bar{B}_{m}(a x) \quad(a \in \mathbb{N}, x \in \mathbb{R}),
$$

which follows easily from (1) and trivially from (2).

3. The function $\beta(u, X)$. We define this by the formula

$$
\beta(u, X)=\sum_{m=0}^{\infty} \frac{\bar{B}_{m}(u)}{m !} X^{m-1} \quad(X \neq 0),
$$

where $u \in \mathbb{R} / \mathbb{Z}$ and we think of $\beta(u, X)$ either as a formal Laurent series (with values in $X^{-1} \mathbb{R}[[X]]$ ) or as a convergent Laurent series for $X$ in the $\operatorname{disc} D=\{X \in \mathbb{C}:|X|<2 \pi\}$. We denote by $(\mathbb{R} / \mathbb{Z})_{0}^{3}$ (respectively $D_{0}^{3}$ ) the set of triples with sum zero in the circle group (respectively disc). The components of such a triple will always be indexed by a number $j \in \mathbb{Z} / 3 \mathbb{Z}$.

The next proposition is the key result of the paper, from which the main theorem follows easily. We give three proofs since each is very short and illustrates a method which is useful in many other calculations of this type.

Proposition. For $\left(u_{1}, u_{2}, u_{3}\right) \in(\mathbb{R} / \mathbb{Z})_{0}^{3}$ and $\left(X_{1}, X_{2}, X_{3}\right) \in D_{0}^{3}$ we have

$$
\sum_{j(\bmod 3)} \beta\left(u_{j}, X_{j+1}\right) \beta\left(-u_{j+1}, X_{j}\right)= \begin{cases}1 / 4 & \text { if } u_{1}=u_{2}=u_{3}=0 \\ 0 & \text { otherwise }\end{cases}
$$

Proof 1. By the definition (1) of $B_{m}(u)$, we have

$$
\beta(u, X)= \begin{cases}\frac{1}{2} \cdot \frac{e^{X}+1}{e^{X}-1} & (u \in \mathbb{Z}), \\ \frac{e^{(u-[u]) X}}{e^{X}-1} & (u \notin \mathbb{Z}),\end{cases}
$$

so the Proposition can be checked by elementary algebra, assuming that each $u_{j}$ lies in $[0,1)$ and distinguishing the cases when none, one, or all three of them are equal to zero. (The last case is just the addition law for the cotangent function.)

Pr o of 2. A second method is to use equation (2). This gives

$$
\begin{aligned}
\beta(u, X) & =\frac{1}{X}-\sum_{m=1}^{\infty} \frac{X^{m-1}}{(2 \pi i)^{m}} \sum_{k \in \mathbb{Z}}^{\prime} \frac{\mathbf{e}(k u)}{k^{m}}=\sum_{k \in \mathbb{Z}} \frac{\mathbf{e}(k u)}{X-2 \pi i k} \\
& =\sum_{\lambda \equiv X(\bmod 2 \pi i)} \frac{e^{(X-\lambda) u}}{\lambda},
\end{aligned}
$$

where the last sum, obtained by writing $\lambda=X-2 \pi i k$, is to be interpreted as a Cauchy principal value (sum over $|\lambda|<L$ and let $L \rightarrow \infty$ ) for convergence. 
This gives

$$
\beta\left(u_{1}, X_{2}\right) \beta\left(-u_{2}, X_{1}\right)=\sum_{\lambda_{1} \equiv X_{1}, \lambda_{2} \equiv X_{2}(\bmod 2 \pi i)} \frac{e^{\left(X_{2}-\lambda_{2}\right) u_{1}-\left(X_{1}-\lambda_{1}\right) u_{2}}}{\lambda_{1} \lambda_{2}} .
$$

Now, setting $\lambda_{3}=-\lambda_{1}-\lambda_{2}$, one has

(i) $\lambda_{j} \equiv X_{j}(\bmod 2 \pi i)$ for all $j \in \mathbb{Z} / 3 \mathbb{Z}$,

(ii) the expression $\exp \left(\left(X_{j+1}-\lambda_{j+1}\right) u_{j}-\left(X_{j}-\lambda_{j}\right) u_{j+1}\right)$ is independent of $j$, and

(iii) $\sum_{j \in \mathbb{Z} / 3 \mathbb{Z}} 1 / \lambda_{j} \lambda_{j+1}=0$.

Therefore the triple sum over all $\left(\lambda_{1}, \lambda_{2}, \lambda_{3}\right) \in \mathbb{C}_{0}^{3}$ with $\lambda_{j} \equiv X_{j}(\bmod 2 \pi i)$ vanishes termwise unless $u_{1}=u_{2}=u_{3}=0$, in which case the Cauchy principal values do not match up and the limit as $L \rightarrow \infty$ must be computed as the Riemann sum

$$
\lim _{L \rightarrow \infty}\left(3 \sum_{\left|\lambda_{1}\right|,\left|\lambda_{2}\right|<L,\left|\lambda_{1}+\lambda_{2}\right|>L} \frac{1}{\lambda_{1} \lambda_{2}}\right)=\frac{6}{(2 \pi i)^{2}} \int_{0}^{1} \int_{1-y}^{1} \frac{d x}{x} \frac{d y}{y}=-\frac{1}{4} .
$$

Pro of 3. Finally, for $X_{j}$ real - it is obviously enough to treat this casewe can use the formula for summing geometric series to rewrite (4) as

$$
\beta(u, X)=\sum_{\kappa \equiv u(\bmod 1)} \delta(X, \kappa) e^{\kappa X} \quad(X \in \mathbb{R}, X \neq 0),
$$

where $\delta(X, \kappa)=\frac{1}{2}(\operatorname{sign}(X)-\operatorname{sign}(\kappa))$. The desired identity then follows by summing over triples of real numbers $\kappa \in \mathbb{R}_{0}^{3}$ which reduce to $u$ modulo 1 and noting that $\exp \left(\kappa_{j} X_{j+1}-\kappa_{j+1} X_{j}\right)$ is independent of $j$ and that $\sum_{j} \delta\left(\kappa_{j}, X_{j+1}\right) \delta\left(-\kappa_{j+1}, X_{j}\right)$ equals $1 / 4$ if $\kappa=(0,0,0) \in \mathbb{R}_{0}^{3}$ and 0 otherwise.

Remark. In the second and third proofs we used the fact that if $\mathbf{x}$ and $\mathbf{y}$ are two triples with sum 0 , then $\Delta_{j}=x_{j} y_{j+1}-x_{j+1} y_{j}$ is independent of $j \in \mathbb{Z} / 3 \mathbb{Z}$. This can be seen by direct calculation or by noting that the $3 \times 3$ determinant $|\mathbf{x} \mathbf{y} \mathbf{z}|=\sum_{j} \Delta_{j} z_{j+2}$ must vanish if the triple $\mathbf{z}$ also has sum 0 .

4. The reciprocity theorem. We now introduce the main object of study, the generalized Dedekind-Rademacher sum

$$
\begin{array}{r}
S_{m, n}\left(\begin{array}{lll}
a & b & c \\
x & y & z
\end{array}\right)=\sum_{h(\bmod c)} \bar{B}_{m}\left(a \frac{h+z}{c}-x\right) \bar{B}_{n}\left(b \frac{h+z}{c}-y\right) \\
(a, b, c \in \mathbb{N}, x, y, z \in \mathbb{R} / \mathbb{Z}) .
\end{array}
$$

Clearly

$$
S_{m, n}\left(\begin{array}{ccc}
k a & k b & k c \\
x & y & z
\end{array}\right)=k S_{m, n}\left(\begin{array}{ccc}
a & b & c \\
x & y & z
\end{array}\right)
$$


so we may (and will) assume that the integers $a, b$, and $c$ have no common factor. Note also that $S_{m, n}\left(\begin{array}{lll}a & b & c \\ x & y & z\end{array}\right)$ depends only on $a$ and $b$ modulo $c$. We want to prove that these sums, which generalize the classical Dedekind sums and their generalizations by Rademacher, satisfy certain reciprocity relations under (simultaneous) cyclic permutations of $(a, b, c)$ and $(x, y, z)$. However, these reciprocity relations mix various pairs of indices $(m, n)$, so they are most conveniently stated in terms of the generating function

(6) $\mathfrak{S}\left(\begin{array}{lll}a & b & c \\ x & y & z \\ X & Y & Z\end{array}\right)=\sum_{m, n \geq 0} \frac{1}{m ! n !} S_{m, n}\left(\begin{array}{ccc}a & b & c \\ x & y & z\end{array}\right)(X / a)^{m-1}(Y / b)^{n-1}$.

Here $X$ and $Y$ are non-zero variables (either formal or else complex of small absolute value) and the variable $Z$, which does not appear explicitly on the right-hand side of the definition, is defined as $-X-Y$. The reciprocity theorem is then:

TheOREM. Let $a, b, c$ be three positive integers with no common factor, $x, y, z$ three real numbers, and $X, Y, Z$ three variables with sum zero. Then

$$
\begin{aligned}
\mathfrak{S}\left(\begin{array}{lll}
a & b & c \\
x & y & z \\
X & Y & Z
\end{array}\right)+\text { cyclic permutations } \\
\quad= \begin{cases}1 / 4 & \text { if }(x, y, z) \in(a, b, c) \mathbb{R}+\mathbb{Z}^{3}, \\
0 & \text { otherwise, }\end{cases}
\end{aligned}
$$

where the sum on the left is over the three terms obtained by cyclic permutation of the columns of the argument of $\mathfrak{S}$.

Remark. Since clearly $\mathfrak{S}$ is symmetric in its first two columns, the left-hand side of (7) is invariant under all permutations of the columns, not just the cyclic ones.

Proof of Theorem. Using the property (3) of $\bar{B}_{m}$, we can rewrite the definition of $S_{m, n}$ as

$$
\begin{aligned}
& a^{1-m} b^{1-n} S_{m, n}\left(\begin{array}{lll}
a & b & c \\
x & y & z
\end{array}\right) \\
& =\sum_{f(\bmod a)} \sum_{g(\bmod b)} \sum_{h(\bmod c)} \bar{B}_{m}\left(\frac{z+h}{c}-\frac{x+f}{a}\right) \bar{B}_{n}\left(\frac{z+h}{c}-\frac{y+g}{b}\right)
\end{aligned}
$$

and hence, setting $\xi=(x+f) / a, \eta=(y+g) / b$ and $\zeta=(z+h) / c$,

$$
\mathfrak{S}\left(\begin{array}{ccc}
a & b & c \\
x & y & z \\
X & Y & Z
\end{array}\right)=\sum_{\substack{(\xi, \eta, \zeta) \in(\mathbb{R} / \mathbb{Z})^{3} \\
(a \xi, b \eta, c \zeta)=(x, y, z)}} \beta(\zeta-\xi, X) \beta(-\eta+\zeta, Y) .
$$

The Theorem now follows immediately from the Proposition of Section 3. 
5. Explicit formulas. By comparing the homogeneous parts of degree $N-2$ in $X, Y$ and $Z=-X-Y$ in the two sides of (7), we obtain for each integer $N \geq 0$ a reciprocity law for the sums (5) with $m+n=N$. For $N=0$ this identity is the trivial $1 /(X Y)+1 /(Y Z)+1 /(Z X)=0$ and for $N=1$ it is vacuous, but for $N=2$ we obtain Berndt's generalization of the classical Dedekind-Rademacher reciprocity law and for higher $N$ we get a whole series of higher reciprocity identities for the sums (5). The word "reciprocity" is used because if (say) $c=1$ then only two of the three terms on the left-hand side of (7) are non-trivial and they are obtained from one another by interchanging the roles of $(a, x, X)$ and $(b, y, Y)$.

As an illustration, we take the case $N=4$. If we multiply both sides of (7) by $X Y Z$, then both sides become power series in $X$ and $Y$, and we must compare the terms of degree 5 . This gives six identities, one for the coefficient of each monomial $X^{i} Y^{5-i}$. Of these, three are trivial and the other three are obtained by permutation of the indices, so there is essentially one reciprocity law. The trivial relations, obtained from the specializations $X=0, Y=0$ and $X+Y=0$, concern the sums $S_{4,0}$ and $S_{4,0}$ and say that

$$
\frac{1}{a^{3}} S_{4,0}\left(\begin{array}{ccc}
a & b & c \\
x & y & z
\end{array}\right)=\frac{1}{c^{3}} S_{4,0}\left(\begin{array}{ccc}
c & b & a \\
z & y & x
\end{array}\right)
$$

and similarly for cyclic permutations. They are trivial because we can evaluate any sum $S_{m, 0}$ or $S_{0, n}$ directly from (3):

$$
S_{m, 0}\left(\begin{array}{ccc}
a & b & c \\
x & y & z
\end{array}\right)=\frac{(a, c)^{m}}{c^{m-1}} \bar{B}_{m}\left(\frac{a z-c x}{(a, c)}\right) .
$$

The non-trivial reciprocity law relates the sums $S_{2,2}$ to the sums $S_{3,1}$ and $S_{1,3}$. Specifically, if we introduce the abbreviations

$$
\begin{aligned}
& \widetilde{S}_{2,2}\left(\begin{array}{lll}
a & b & c \\
x & y & z
\end{array}\right)=\frac{1}{4 a b} S_{2,2}\left(\begin{array}{lll}
a & b & c \\
x & y & z
\end{array}\right) \\
& \widetilde{S}_{3,1}\left(\begin{array}{lll}
a & b & c \\
x & y & z
\end{array}\right)=\frac{1}{6 a^{2}}\left[S_{3,1}\left(\begin{array}{lll}
a & b & c \\
x & y & z
\end{array}\right)+S_{3,1}\left(\begin{array}{lll}
a & c & b \\
x & z & y
\end{array}\right)\right]
\end{aligned}
$$

(so that $\widetilde{S}_{2,2}$ is symmetric in its first two and $\widetilde{S}_{3,1}$ in its last two columns), then (7) and (8) give

$$
\begin{aligned}
\widetilde{S}_{2,2}\left(\begin{array}{lll}
a & b & c \\
x & y & z
\end{array}\right)= & \widetilde{S}_{3,1}\left(\begin{array}{lll}
a & b & c \\
x & y & z
\end{array}\right)+\widetilde{S}_{3,1}\left(\begin{array}{ccc}
b & a & c \\
y & x & z
\end{array}\right) \\
& -\frac{a(b, c)^{4}}{24 b^{3} c^{3}} \bar{B}_{4}\left(\frac{b z-c y}{(b, c)}\right)-\frac{b(a, c)^{4}}{24 a^{3} c^{3}} \bar{B}_{4}\left(\frac{a z-c x}{(a, c)}\right) \\
& -\frac{c(a, b)^{4}}{8 a^{3} b^{3}} \bar{B}_{4}\left(\frac{a y-b x}{(a, b)}\right)
\end{aligned}
$$


or, equivalently,

$$
\begin{aligned}
2 \widetilde{S}_{3,1}\left(\begin{array}{lll}
a & b & c \\
x & y & z
\end{array}\right) & =\widetilde{S}_{2,2}\left(\begin{array}{lll}
a & b & c \\
x & y & z
\end{array}\right)+\widetilde{S}_{2,2}\left(\begin{array}{lll}
a & c & b \\
x & z & y
\end{array}\right)-\widetilde{S}_{2,2}\left(\begin{array}{lll}
b & c & a \\
y & z & x
\end{array}\right) \\
& -\frac{a(b, c)^{4}}{24 b^{3} c^{3}} \bar{B}_{4}\left(\frac{b z-c y}{(b, c)}\right)+\frac{b(a, c)^{4}}{8 a^{3} c^{3}} \bar{B}_{4}\left(\frac{a z-c x}{(a, c)}\right) \\
& +\frac{c(a, b)^{4}}{8 a^{3} b^{3}} \bar{B}_{4}\left(\frac{a y-b x}{(a, b)}\right) .
\end{aligned}
$$

In general, if we write $\mathfrak{S}_{+}$for the subsum of (6) with $m$ and $n$ strictly positive (so that $\mathfrak{S}_{+}$is a power series rather than a Laurent series in $X$ and $Y$ ), then (8) shows that the left-hand side of equation (7) equals

$$
\begin{gathered}
\mathfrak{S}_{+}\left(\begin{array}{ccc}
a & b & c \\
x & y & z \\
X & Y & Z
\end{array}\right)+\sum_{m=2}^{\infty} \frac{(a, c)^{m} b}{m ! a^{m-1} c^{m-1}} \bar{B}_{m}\left(\frac{a z-c x}{(a, c)}\right) \frac{X^{m-1}-(X+Y)^{m-1}}{Y} \\
+ \text { cyclic permutations }
\end{gathered}
$$

which is again a power series in $X$ and $Y$. Comparing the coefficients of degree $N-2$ of this and the right-hand side of (7) we get $N-1$ relations among $3(N-1)$ non-trivial Dedekind-Rademacher sums $S_{m, n}(m+n=N$, $m, n \geq 1$ ) and these, together with the evident symmetry between $S_{m, n}$ and $S_{n, m}$, let us express all the sums $S_{m, n}$ as linear combinations of those with $m>2 n+O(1)$.

Acknowledgements. We are indebted to the UK Science and Engineering Research Council for the award of a Research Studentship to Julie Wilson.

\section{References}

[1] T. M. Apostol, Generalized Dedekind sums and the transformation formulae of certain Lambert series, Duke Math. J. 17 (1950), 147-157.

[2] B. C. Berndt, Reciprocity theorems for the Dedekind sums and generalizations, Adv. in Math. 23 (1977), 285-316.

[3] L. Carlitz, Some theorems on generalized Dedekind sums, Pacific J. Math. 3 (1953), $513-522$.

[4] -, A three-term relation for Dedekind-Rademacher sums, Publ. Math. Debrecen 14 (1967), 119-124.

[5] R. Dedekind, Erläuterungen zu den Fragmenten XXVIII, in: B. Riemann's Gesammelte mathematische Werke, 2nd ed., Teubner, Leipzig, 1892, 466-478.

[6] R. R. Hall and J. C. Wilson, On reciprocity formulae for inhomogeneous and homogeneous Dedekind sums, Math. Proc. Cambridge Philos. Soc. 114 (1993), 9-24. 
[7] M. Mikolás, On certain sums generating the Dedekind sums and their reciprocity laws, Pacific J. Math. 7 (1957), 1167-1178.

[8] J. L. Raabe, Zurückführung einiger Summen und bestimmten Integrale auf die Jacob-Bernoullische Funktion, J. Reine Angew. Math. 42 (1851), 348-367.

[9] H. Rademacher, Generalization of the reciprocity formula for Dedekind sums, Duke Math. J. 21 (1954), 391-397.

[10] H. Rademacher and E. Grosswald, Dedekind Sums, Carus Math. Monographs 16, Math. Assoc. Amer., 1972.

[11] L. Takács, On generalized Dedekind sums, J. Number Theory 11 (1979), 264-272.

[12] J. C. Wilson, D. Phil. Thesis, University of York, United Kingdom, 1993.

DEPARTMENT OF MATHEMATICS

UNIVERSITY OF YORK

YORK YO1 5DD, UNITED KINGDOM

\author{
MAX-PLANCK-INSTITUT FÜR MATHEMATIK \\ GOTTFRIED-CLAREN-STRASSE 26 \\ 53225 BONN, GERMANY
}

Received on 9.3.1995

and in revised form on 15.3.1995 